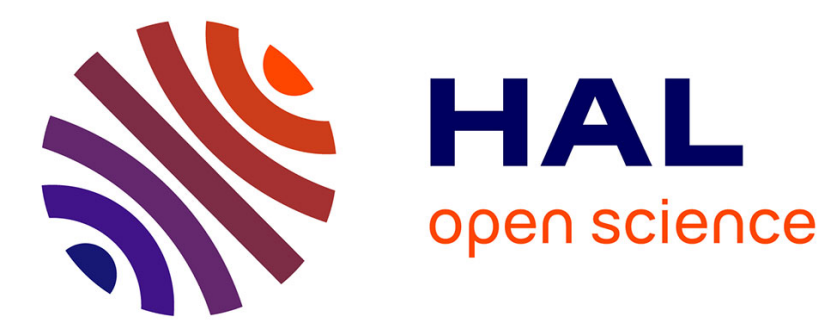

\title{
La politique d'aménagement musical du territoire de Marcel Landowski
}

Noémi Lefebvre

\section{To cite this version:}

Noémi Lefebvre. La politique d'aménagement musical du territoire de Marcel Landowski. 2009. halshs-00437039

\section{HAL Id: halshs-00437039 \\ https://shs.hal.science/halshs-00437039}

Preprint submitted on 10 Dec 2009

HAL is a multi-disciplinary open access archive for the deposit and dissemination of scientific research documents, whether they are published or not. The documents may come from teaching and research institutions in France or abroad, or from public or private research centers.
L'archive ouverte pluridisciplinaire HAL, est destinée au dépôt et à la diffusion de documents scientifiques de niveau recherche, publiés ou non, émanant des établissements d'enseignement et de recherche français ou étrangers, des laboratoires publics ou privés. 
Noémi Lefebvre

I.E.P. de Grenoble

P.A.C.T.E.

Séminaire P.A.C.T.E "Culture(s) et constructions symboliques des territoires"

Communication au colloque "Musique, territoire et développement local", Cité des Terrioires, Institut de Géographie Alpine, 19 et 20 novembre 2009

\section{La politique d'aménagement musical du territoire de Marcel Landowski.}

la politique de la musique engagée par Marcel Landowski en 1966 poursuit l'objectif annoncé de "rendre la musique accessible à tous", par "l'implantation progressive de centres musicaux de premier ordre en différents points du territoire", exigeant une réorganisation des structures professionnelles d'enseignement et de diffusion ${ }^{1}$. Elle organise la réforme des structures musicales sur lesquelles s'appuira le développement de "régions musicales", conçu comme un développement musical en région à partir d'un modèle central.

Formalisée en 1969 par le "plan de dix ans pour l'organisation des structures de la musique" cette politique se réfère aux grandes lignes de la politique de l'aménagement du territoire inaugurée en 1960 avec la délimitation des Régions comme "circonscriptions d'action régionale", et développée à partir de 1963 par la DATAR. La participation de la politique de diffusion musicale à l'aménagement du territoire offre à ce plan une base administrative, lui donne sens par rapport à un contexte plus large, et permet de l'inscrire dans la modernité.

Principes et pragmatique constituent deux aspects complémentaires d'une action qui suscite un fort mouvement d'adhésion de la part des élus comme des musiciens. De principe égalitaire, le plan de dix ans organise une décentralisation sans diversification, soucieuse d'équilibre géographique et de rayonnement local, mais dès 1966, la présence de ressources musicales institutionnnelles dessine les priorités. La réforme structurelle implique un mode d'action qui tienne compte de ce terrain musical. Elle impose, quitte à accentuer provisoirement un déséquilibre entre les régions, un rapport étroit de la direction de la musique avec les collectivités locales et les institutions musicales existantes, implication que Marcel Landowski organise en développant l'animation musicale dans quelques régions exemplaires, à partir desquelles doit se modéliser la politique nationale.

La politique d'animation s'inspire d'abord de l' "action culturelle" dont elle reprend certaines préoccupations - toucher un nouveau public, permettre la rencontre directe de celui-ci avec les grandes œuvres -, selon le principe d'accès de tous à la musique "de haute qualité", dont personne ne discute alors les critères.

A partir de 1969, dans le cadre du "plan de dix ans", elle est associée à une "insfrastructure administrative et technique", envisagée à la fois comme un moyen de représentation, au plan régional et départemental, du service de la musique, une courroi de

${ }^{1}$ Cette politique est annoncée pour la premieère fois et de manière très claire dans une Note sur la musique, Rapport de Monsieur Landowski, Inspecteur général de l'enseignement musical, à l'intention de Monsieur Antoine Bernard, Directeur du Cabinet de Monsieur le Ministre des Affaires Culturelles, datée du 21 avril 1966.

(A.N.19950514/24 -1) 
transmission entre Paris et le territoire, et comme une étape dans la construction d'une "décentralisation culturelle" dans le mouvement de la création des premières D.R.A.C.

\section{L'accès de tous à la musique véritable}

Lorsque débute, au sein du ministère des Affaires culturelles, la réflexion sur l'action culturelle appliquée à la musique, la "mise en présence des œuvres capitales de l'humanité" au "plus grand nombre de Français" en constitue le principe général. Ce "plus grand nombre", formule performative du décret fondateur de $1959^{2}$, renvoie à l'objectif essentiel de démocratisation, lequel se traduit, dans ce domaine spécifié de la culture, par une préoccupation portée sur le public.

S'agissant de la musique, ce public, défini traditionnellement comme l'ensemble des individus réunis dans une salle de concert, est devenu de plus en plus abstrait et insaisissable : "la radio et le disque se sont substitués à la présence vivante des orchestres, et même des vedettes; d'où il s'ensuit qu'un dialogue s'est instauré, au travers les espaces, entre deux partenaires sans visage", ${ }_{3}$ analyse Emile Biasini, directeur des théâtres, de la musique et de l'action culturelle.

Quand la diffusion des œuvres n'est plus localisable, l'incertitude concerne autant la pérénité de "l'œuvre d'art à l'époque de sa reproduction mécanisée"” que l'identification de ces inquiétants "visages invisibles".

Le terme de public implique un rapport plus ou moins occasionnel ou stable à une œuvre; dans le contexte d'une politique musicale égalitaire, cet ensemble social défini par une disposition l'égard d'un art doit correspondre idéalement à toute la nation, ainsi s'étendre à tout le territoire national. La préoccupation n'est pas avant tout sociale, mais bien géographique. Il faut d'abord qu'il y ait de la musique partout, pour que tous les Français puissent y accéder.

\section{Le projet d'action musciale dans le rapport de la commission d'études des problèmes de la musique.}

La réunion, décidée par Malraux, d'une commission d'études pour les problèmes de la musique $^{5}$, donne l'occasion à Emile Biasini de développer un projet d'action musicale dans le contexte du rapport d'expertise que doit produire cette commission.

Contrairement à l'action culturelle lancée avec les maisons de la culture à partir du théâtre en 1962, l'action musicale n'est pas, avant la création du service de la musique, le résultat

\footnotetext{
${ }^{2}$ Decret $n^{\circ} 59-889$ du 24 juillet 1959 : "Le ministère chargé des affaires culturelles a pour mission de rendre accessibles les œuvres capitales de l'humanité, et d'abord de la France, au plus grand nombre possible de Français ; d'assurer la plus vaste audience à notre patrimoine culturel, et de favoriser la création des œuvres d'art et l'esprit qui l'enrichissent"

${ }_{3}$ Rapport de la Commission nationale pour l'étude des problèmes de la muisique, 1963-1964, miistère d'Etat des affaire culturelles, p.12.

${ }^{4}$ Titre de l'essai de Walter Benjamin publié en 1935,Das Kunswerk im Zeitalter seiner technischen Reproduzierbarkeit, publication en français de la première version de l'article, en 1936, dans la traduction de Pierre Klossowski.

${ }^{5}$ Arrêté du 27 décembre 1962, portant institution d'une commission chargée d'étudier les problèmes de la musique en France et d'élaborer dans ce domaine un plan d'action conforme à la mission générale du ministère d'État chargé des Affaires culturelles (.J.O. 29 décembre 1962).
} 
d'une politique publique, mais désigne un ensemble d'actions principalement menées par les fédérations musicales. ${ }^{6}$ Pour que l'action musicale devienne une véritable politique culturelle, il s'agit que ces initatives trouvent un mode de rassemblement autour d'objectifs publics clairement établis, prioritairement dans ces lieux à vocation polyvalente que sont les Maisons de la culture.

L'action musicale dépasse cependant ce seul cadre des Maisons de la culture : Emile Biasini propose de mener une expérience dans quelques "villes témoin", choisies pour leur situation géographique, démographique, sociale et artistique, où l'activité musicale y serait coordonnée, avec la participation de tous les moyens de l'Etat : RTF, décentralisation lyrique, maisons de la culture, etc. Ces"villes-témoin", conçues comme sorte de laboratoire permettant d'obtenir "des renseignements sociaux, d'établir des statistiques, de susciter des éléments de réponse aux diverses questions soulevées", serviraient de base de départ, de lieux d'impulsion exemplaires à partir desquels l'expérience pourrait se généraliser.

L'idée se transforme, dans le rapport final de la Commission d'études, en projet de création de "cités pilotes", l'expression contenant une dimension futuriste que n'avait pas la "ville témoin". Les "cités-pilotes" allient à la fois l'imaginaire de la cité utopique et l'action culturelle expérimentée dans les maisons de la culture.

Le problème que pose la réalisation de ce programme réside cependant bien dans la "reconversion" d'un milieu musical professionnel à l'animation culturelle : là réside l'imaginaire de ces "cités pilote", selon Pierre Schaeffer ${ }^{7}$, rapporteur à la commission du plan en 1965 :

"Que sont en effet ces “cités-pilotes" musicales, lorsqu'on sait combien sont précis les problèmes d'animation culturelle et quelles compétences nouvelles il faut former pour $\mathrm{y}$ répondre. Si l'on disait que quelques milliers de musiciens formés comme animateurs allaient être reconvertis dans ces secteurs d'animation (discothèques, maisons de la musique, organisation des loisirs, etc...), nous trouverions un caractère réaliste à ce qui, pour le moment, n'en a pas dans la brièveté du rapport. (...) [c'est]tout le problème des loisirs culturels, sociaux ou de jeunesse, que le rapport ne fait qu'évoquer de façon imaginaire et bien peu sérieuse dans ce qu'il appelle "cités-pilotes" .

Le terme de "reconvertis" choisi par Pierre Schaeffer montre à quel point le projet de "cités pilotes", dont il salue la nouveauté, est caractéristique de la distance entre cette politique "inventée" qu'est la politique d'action culturelle et les préoccupations des musiciens professionnels dans les années soixante.

La mise à distance, par le chef de la D.T.M.A.C., de la profession musicale et des institutions qui l'ont constituée et la font vivre, est le corrolaire d'un projet où la mise en

\footnotetext{
"Il n'y a pas d'action musicale absolue, s'adressant au plus grand nombre pour le plus grand bien de la musique, mais une multitude d'actions particulières, généreuses mais souvent intolérantes, et limitées dans leur objectif du fait même de leur extrême spécialisation" constate Emile Biasini dans le Rapport de la commission nationale pour l'étude des problèmes de la musique,1963-1964, Ministère d'Etat Affaires Culturelles, 131p., p.68

${ }^{7}$ Pierre Schaeffer (1912-1995) fondateur du Groupe de recherches musicales,G.R.M., dirige alors le Service de la recherche de la R.T.F.

${ }^{8}$ Déclaration de Pierre Schaeffer à la commission du plan, 5 juin 1965.

${ }^{9}$ La pertinence du titre de l'ouvrage de Philippe Urfalino, L'invention de la politique culturelle,(Paris, Pluriel, 2004) est ici particulièrement évidente.
} 
présence du public avec les œuvres est pensée en dehors d'une implication traditionnelle de l'Etat dans l'organisation et le contrôle de la musique ${ }^{10}$, et dont l'héritier le plus légitime, pour la profession comme pour le Ministre, est l'Inspecteur de la musique, attaché au Service des enseignement artistiques du ministère, administrativement séparé de l'action culturelle.

\section{L'animation musicale reprise par Marcel Landowski.}

Le choix, opéré par le ministre en 1966, de détacher la politique musicale de l'action culturelle pour la confier à Marcel Landowski, Inspecteur de la musique nommé en décembre 1964, redéfinit les modalités de cette politique au plan territorial : l'aménagement musical du territoire reposera d'abord sur la rénovation, dans chaque région, des structures d'enseignement et de diffusion qui constitueront les centres de rayonnement musical régional.

Pour développer une politique qui soit, dans le mouvement de l'Aménagement du territoire, basé sur les Régions, Marcel Landowski a besoin de correspondants, hommes de confiance capables d'impliquer aussi bien les élus que les musiciens dans sa réalisation, et de faire remonter au Service de la musique des informations sur les situations, les avancées ou les difficultés rencontrées. Or, en 1966, un tel relais n'existe pas. ${ }^{11}$

Marcel Landowski s'appuie alors sur l'expérience réussie de l'action culturelle ${ }^{12}$, consistant à confier à des "animateurs" la rencontre des œuvres avec le public.

Dès l'été 1966, il organise ainsi une politique d'animation musicale différenciée de la politique d'action culturelle ${ }^{13}$. Les animateurs musicaux seront chargés de développer la vie musicale dans une région en y assurant la coordination des activités musicales et en suscitant des activités nouvelles.

\section{Le recrutement des premiers animateurs musicaux en région}

Le profil de ces animateurs-correspondants du Service de la musique se dessine à partir d'une double compétence. Leur formation administrative s'effectuera par la pratique, selon la logique qu'il est plus aisé de former un musicien professionnel cultivé aux rudiments de

\footnotetext{
${ }^{10}$ Cf. Noémi Lefebvre, "Le modèle français d'enseignement musical", in : Enseigner la musique, Actes des Journées d'études sur l'avenir de l'enseignement spécialisé de la musique, Lyon, CEFEDEM Rhône-Alpes, DRAC Rhône-Alpes, F.N.C.C., Observatoire des Politiques Culturelles, 2002, t. 1, p. $43-56$.

11 'Les seuls relais possibles que j'aie trouvés en 1966 étaient les quarante-quatre conservatoires municipaux contrôlés, aidés par l'Etat, et leurs directeurs, leurs professeurs. Encore faut-il ajouter que près de soixante départements n'en possédaient aucun!" Marcel Landowski, Batailles pour la musique, Paris, Seuil, 1979, 192p. p.122.

${ }^{12}$ Marcel Landowski prend soin, dès 1964, de montrer en permanence une attitude ouverte à l'égard de la politique d'action culturelle, à laquelle est identifié, même après le départ de Gaëtan Picon et d'Emile Biasini en 1966 , et ce jusqu'à l'inflexion de 1968, le Ministère des Affaires Culturelles. Il ne conçoit pas que le Service de la musique puisse se construire et construire une politique "contre" l'action culturelle, mais en dehors de celleci. Ce qui n'est évidememnt pas l'avis d'Emile Biasini.

13 "Si la conception de M.Landowski était si proche de celle da la D.T.M.A.C (M.Biasini), l'antagonisme entre les deux Directions a pourtant éludé toute formation commune et tout critère de recrutement commun pour les animateurs musicaux des Maisons de la Culture et les animateurs musicaux régionaux", souligne Jean-Claude Foulon, animateur musical en région Rhone-Alpes de 1967 à 1972. "Pourtant, dans les faits, nous nous sommes les uns et les autres retrouvés sur le terrain avec nos affinités certaines de musiciens, tout en ayant des missions et des champs d'action différents ; ce qui devait rendre nos « métiers » également différents." (Témoignage de Jean-Claude Foulon, 22 novembre 2008)
} 
l'administration publique, que de faire d'un administrateur qualifié un musicien accompli. Ce "nouveau type d'homme-musicien"" sera, à l'image de Marcel Landowski, un musicien d'abord et un administrateur par occasion, capable de comprendre ces deux langages pour les traduire en analyses, en projets, en actions et résultats.

Ce nouveau métier intéresse de jeunes musiciens motivés par l'engagement social et l'action politique, mais aussi, plus prosaïquement, en quête d'un emploi.

Après ses études au conservatoire, son prix de Rome, ses cours de direction d'orchestre avec Pierre Boulez à Bâle, inscrit dans la classe de Messiaen, ayant suivi les cours de Stockhausen à Cologne, Michel Decoust a trente ans et se trouve sans travail et sans ressources. En mai 1966, il découvre en lisant "Le Monde"15 le "non" retentissant de Pierre Boulez à Malraux suite à la nomination de Marcel Landowski à la tête d'un Service de la musique. Il apprend que celui-ci veut faire appel à des "défricheurs de terrain". Aussitôt, il se porte volontaire.

“J'ai toujours eu la volonté d'être un constructeur, avec une responsabilité sociale et humaine. J'ai été le voir, il m'a reçu, il me connaissait parce que je faisais partie des dix-quinze compositeurs du moment. Je lui ai dit que j'étais prèt à partir en province..."16

Jean-Claude Foulon a accumulé les premiers prix au C.N.S.M. et se retrouve, jeune compositeur, devant un avenir professionnel à inventer, quand le Service de la musique fait savoir que des postes d'animateur seront créés :

"Ma première entrevue avec Marcel Landowski eut lieu en Juin 1966, sur la recommandation de Raymond-Gallois Montbrun, directeur du C.N.S.M. de Paris, lequel me servit d'orienteur vers une activité professionnelle la fin de mes études ; j'étais dans ma 27e année"

Entre décembre 1966 et mai 1967, trois stages sont organisés, à la fondation culturelle de Royaumont où interviennent des psychologues, sociologues et spécialistes de la communication, et à l'issue desquels six animateurs sont retenus.

Leur mission n'est pas précisément établie, mais s'organise à la fois en fonction des objectifs prioritaires du Service de la musique dans les régions choisies, des situations de terrain et des profils et motivations des animateurs retenus.

Sans reprendre strictement l'idée de "cités pilotes", Marcel Landowski concentre d'abord sa politique d'animation, en parallèle avec la restructuration de la diffusion, sur quelques régions bénéficiant, soit déjà d'une activité de diffusion musicale importante autour des grandes villes, Paris bien sûr, mais aussi Lyon et Reims, soit d'un potentiel de développement important, comme en Pays de Loire, en rapprochant les activités des orchestres d'Angers et de Nantes. Les résultats obtenus permettront d'étendre progressivement le modèle à tout le territoire.

\footnotetext{
${ }^{14}$ Marcel Landowski, Séance du 14 juin 1972 à l'Académie des Beaux-Arts

15 "M. Marcel Landowski vient d'être nommé à la direction de la musique qui vient d'être créée par M. Malraux", in : Le Monde du 4 mai1966, p.8. "Pierre Boulez "dit non à Malraux" ”, in : Le Monde du 26 mai 1966, p.14.

${ }^{16}$ Témoignage de Michel Decoust,17 février 2006.
} 
Jean-Paul Rieunier, ancien élève du C.N.S.M. et compositeur, et Michel Briguet, venu des Jeunesses Musicales de France et "musicographe"17, sont affectés à la Région parisienne, Jacques Roussel, formé au conservatoire d'Innsbruck et au C.N.S.M. est affecté en Ile de France. Jacques Herbillon, élève au Conservatoire de Reims, puis à l'Ecole Normale de musique et au Conservatoire de Genève, est affecté en Champagne-Ardennes, sa région d'origine. JeanClaude Foulon est affecté en région Rhône-Alpes et Michel Decoust, dans les Pays de la Loire. Ces deux derniers sont chargés, en plus de l'animation musicale régionale, de "préparer les assises des futurs orchestres régionaux"18 ,éléments fondamentaux d'une démocratisation musicale entendue comme accès à la musique vivante.

\section{Une organisation pragmatique de l'animation musicale en région}

La clarification de la position des animateurs par rapport aux différentes instances qui peuvent en revendiquer la tutelle, municipalités, institutions culturelles, correspondants régionaux du ministère, s'effectue à mesure que se déploie leur activité au plan régional. Bien qu'aucun document officiel ne l'impose ni même ne l'explicite, c'est à Marcel Landowski que rend compte directement chaque animateur musical, et c'est d'abord pour le Service de la musique et en fonction des priorités nationales qu'il agit au plan régional.

Marcel Landowski anticipe cependant la décentralisation culturelle à partir des Régions, et place les animateurs musicaux sous l'autorité du Préfet de Région en attendant la nomination des premiers Directeurs régionaux des Affaires culturelles ${ }^{19}$. L'animateur en région peut ainsi être le vecteur d'une politique musicale nationale que la Région, entité administrative sans statut de collectivité et sans "réalité culturelle"au sens anthropologique du terme, permet de relayer sur le territoire qu'elle circonscrit.

L'échelon régional permet aux animateurs musicaux de déployer leur action en abordant les situations locales sans être partie prenante, mais protégés en quelque sorte de "l'esprit de clocher'à la fois par leur qualité d'expert musical et par leur relation directe avec le chef du Service de la musique. L'animateur musical participe ainsi à cet aménagement culturel du territoire à partir des régions tout en étant "porte-parole de la politique suivie rue SaintDominique". ${ }^{20}$

Telle peut apparaître cette première étape de la décentralisation musicale : appuyée sur six animateurs musicaux sans statut, sans beaucoup de moyens et qui tentent, là où ils sont envoyés, de mettre en œuvre au plan régional une politique définie nationalement d'animation de la diffusion musicale.

Dans la pratique, l'animateur n'est pas seulement le relais d'une politique conçue au niveau central, mais il participe à la connaissance et à la construction de la région musicale : chargé de faire l'inventaire des ressources musicales de la région, il établit en même temps son domaine d'action et sa feuille de route. Ce premier travail topographique que chaque animateur

\footnotetext{
${ }^{17}$ Il est l'auteur de deux ouvrages : Faire de la musique, l'amateur actif et ses problèmes, 1960 et Cinquante millions de Français devant la musique,1965, publiés aux Editions Ouvrières.

${ }_{18}$ Courrier de Marcel Landowski à M.Sauzay, Conseiller Technique, 23 septembre 1968 (A.N.19860732/3)

${ }^{19}$ Les cinq premières D.R.A.C. sont créées en 1969 en Île-de-France, Rhône-Alpes, Alsace, Provence-Alpes-

Côte d'Azur et Corse. Jacques Duhamel et Jacques Rigaud entérinent leur généralisation, effective sous Françoise Giroud, en 1977.

${ }^{20}$ Jean-Claude Foulon, Courrier au Comité d'Histoire du Ministère de la Culture, 21 janvier 2006, p.6.
} 
a commencé par réaliser, constitue en outre la première documentation disponible sur la vie musicale des quatre premières régions concernées, Ile de France, Champagne-Ardennes, Pays de la Loire, et Rhône-Alpes. Ces enquêtes, ni statistiques, ni sociologiques, mais axées sur l'évaluation des activités musicales du point de vue de leur qualité, de leur spécificité, de leurs perspectives de développement, font apparaître l'existence musicale d'un territoire dont la délimitation est alors plus administrative que culturelle.

L'animateur musical "en région"est aussi animateur musical "d'une région": organisateur d'orchestres, promoteur de concerts, initiateur de relations avec les élus, les maisons de la culture, les populations urbaines ou rurales, les publics scolaires, etc. Il est véritablement l'interlocuteur musical en région des musiciens, des élus et des acteurs culturels. ${ }^{21}$

Si Marcel Landowski est lui-même un homme de terrain, n'hésite pas à se déplacer pour rencontrer les élus et les musiciens, il est aussi en relation de confiance avec les animateurs, leur laisse toute initiative une fois le cadre de leur action établi.

\section{Bilans et évolution de l'animation musicale après 1968}

Lancée en 1967, l'animation musicale en région est directement concernée par la mise en cause, en mai 1968, du paradigme de la démocratisation culturelle. La notion de "non-public", développée par Francis Jeanson ${ }^{22}$ " immensité humaine composée de tous ceux qui n'ont encore aucun accès ni aucune chance d'accéder prochainement au phénomène culturel ${ }^{23}$ a été reprise dans la déclaration de Villeurbanne signée par les 23 directeurs de maisons de la culture et théâtres populaires le 25 mai 1968, et constitue une critique sociale et politique de l'organisation de l'accès aux "grandes œuvres". Pour les animateurs musicaux,

"Mai 1968 a été l'occasion d'une pose de réflexion « à chaud », puisque les animateurs régionaux ont été réunis du 18 au 22 juin par la Direction de la Musique, à Royaumont.

Certains animateurs musicaux s'interrogent sur les limites de l'action pour l'élargissement du public de concert, un public composé d'individus dans "l'innocence culturelle" ${ }^{25}$; la rencontre de la musique avec le public n'est pas seulement une question de

\footnotetext{
${ }^{21}$ Cette compétence est jugée par les animateurs eux-mêmes, dans le bilan qu'ils font de leur activité en 1970, comme une absolue nécessité pour mener leur action : "L'animation musicale ne peut être menée que par un musicien professionnel pratiquant, averti des problèmes de gestion ou par une équipe placée sous la responsabilité d'un musicien professionnel pratiquant, averti des problèmes de gestion." (Rapport de stage des animateurs musicaux Royaumont, du 8 au 12 juillet 1970, A.N.19860731/8)

${ }^{22}$ Sur les positions de Francis Jeanson concernant la démocratisation culturelle, cf.Francis Jeanson, L'action culturelle dans la cité, Paris, Seuil,1973.

${ }^{23}$ Déclaration de Villeurbanne du 25 mai 1968. Document de la commission Art et révolution, 25 mai 1968 (B.M. Lyon, collection Jacques Baur, Ms 7053).

${ }^{24}$ Témoignage Jean-Claude Foulon, 22 novembre 2008.

${ }^{25} \mathrm{La}$ formule est de Pierre Gaudibert, et rend compte du postulat anti-éducatif de l'action culturelle qui ne tient pas compte du capital culturel lié aux déterminismes familiaux et scolaires, qui fait obstacle ou facilite l'accès aux œuvres. (Pierre Gaudibert, Action culturelle : intégration et/ou subversion, Tournai, Casterman, $2 \mathrm{e}$ éd. 1973.)
} 
"mise en présence", mais aussi de langage partagé.$^{26}$ La diffusion musicale ne doit plus se concevoir comme une "action de distribution de sens univoque", mais doit prendre en compte la communication, "ce qui implique une culture de participation, une pratique musicale d'interprétation et de création étendue au plus grand nombre d'individus, ce qui ne fait qu'un avec une action d'initiation musicale de base pour tous. ${ }^{, 27}$

La mise en cause de la Culture, critiquée comme institution d'Etat reproductrice d'inégalités sociales ${ }^{28}$, ne provoque pas, de la part du Service de la musique, de réponse rigide ni de repli indigné : les "difficultés nouvelles", explique Marcel Landowski à Alain Trapenard, conseiller technique au Cabinet de Malraux, demandent de mettre en place "sans attendre" une "politique coordonnée" à partir d'une régionalisation musicale selon le modèle des "deux régions exemplaires" ${ }^{29}$ que constituent Rhône-Alpes et les Pays de Loire, les deux régions où l'animation s'est effectuée à partir de la rénovation des orchestres.

\section{L'animation musicale réorganisée par le plan de dix ans.}

Avant même que se précise, suite à mai 1968, puis aux travaux de la commission des affaires culturelles du VIe plan $^{30}$, une inflexion de la politique de démocratisation de l'art vers le "développement culturel", et prenant en compte la création des trois premières D.R.A.C. ${ }^{31}$, processus lancé par Malraux afin de créer "une situation irréversible" dans l'organisation de la culture en région ${ }^{32}$, Marcel Landowski établit, en juillet 1969, un "plan de dix ans pour l'organisation des structures musicales françaises", document de référence d'une politique musicale axée sur le développement de "régions musicales", possédant chacune son conservatoire, son orchestre, son théâtre lyrique ${ }^{33}$ et son animation.

Le plan de dix ans confirme la politique engagée depuis 1966, et introduit un nouvel objectif, inscrit en son premier point : l'organisation de structures administratives, à laquelle est

\footnotetext{
26 “Le public n’a généralement pas la compréhension directe du langage musical et de ce fait -le hiatus avec la pensée des créateurs demeure aussi grand"; - la motivation du public est de plus en plus façonnée selon des critères extra-musicaux (vedettariat, notion de succès dans le spectacle)" (Rapport de stage des animateurs musicaux à Royaumont, du 5 au 8 octobre 1970.(Archive personnelle de Jean-Claude Foulon, rédacteur de ce document.)

${ }^{27}$ Rapport de stage des animateurs musicaux à Royaumont, du 5 au 8 octobre 1970.(Archive personnelle de JeanClaude Foulon, rédacteur de ce document.)

${ }^{28}$ Le livre de Jean Dubuffet, Asphyxiante Culture, publié en 1968, est emblématique de cette association de la culture à l'Etat, à la bourgeoisie et aux académismes, opposée à l'art,libre et libérateur,appartenant à l'individu. (Jean-Jacques Pauvert,1968, réédité chez Minuit, 128p.1986)

${ }^{29}$ Courrier de Marcel Landowski à Alain Trapenard, 17 juillet 1968 ( AN.19860732/3)

${ }^{30}$ Commission des affaires culturelles du VIe Plan, présidée par Pierre Emmanuel : activités, 1970 ; politique de la culture : rapport de P. Emmanuel, novembre 1970. Archives de Georges Pompidou (1962 - 1974) (Centre Beaubourg 774AP/19)

${ }^{31}$ En Alsace, Pays de Loire et Rhône-Alpes, où elles seront dirigées réspectivement par Jean Dumas, André Lebœuf et Claude Hiriart, correspondants permanents du ministère depuis 1963.

${ }^{32}$ Jean-Luc Bodiguel, L'implantation du ministère de la culture en région, Naissance et développement des directions régionales des affaires culturelles, Comité d'histoire du ministère de la culture, Paris, la documentation française, 2000, p 64 et suivantes.

${ }^{33}$ Par le décret 69-297 du 2 avril et arrêté du 26 avril 1969, le Service de la Musique voit sa compétence étendue aux domaines lyrique et chorégraphique. Le service regroupe désormais toutes les actions concernant la musique et relevant du ministère des Affaires culturelles, il est rattaché à une nouvelle Direction, celle des Spectacles, de la Musique et des Lettres.
} 
associée l'activité traditionnelle de "contrôle"dévolue à l'inspection, également régionalisée :

"La mission de l'Etat devant être d'incitation et de contrôle, la mise en place progressive de structures régionales devra reposer auprès des Directeurs Régionaux des Affaires culturelles sur :

-de inspecteurs plurirégionaux (six)

-des délégués musicaux (un par région de programme)

-des animateurs départementaux.(au moins un par département) ${ }^{334}$

Cette structuration administrative de l'animation musicale en région, complétée par un échelon départemental jusqu'alors inexistant, constitue la réponse de Marcel Landowski au constat des limites de la démocratisation musicale et à l'existence de ce "non public" qu'il décrit, positivement, comme "immense public actuellement peu ou pas concerné" au devant duquel il s'agit d'aller. ${ }^{35}$

Ce plan d'organisation de l'animation comme mode d'administration régionale de la musique répond à plusieurs préocupations :

- Une préoccupation égalitaire : le privilège de quelques régions, Rhône-Alpes, Val de Loire, Ile de France et Champagne-Ardenne, ne peut être durablement justifié ; le plan de dix ans permet de faire valoir la nécessité d'étendre cette politique pour mettre fin aux inégalités géographiques. - Une préoccupation d'aménagement régional : l'organisation administrative de l'animation permet une mise en cohérence musicale des régions à partir de structures contrôlées et coordonnées sur le territoire régional. Aussi la région est-elle clairement réaffirmée comme l'espace idéal de mise en cohérence des structures musicales :

"Pour animer et contrôler la qualité et l'action des divers élément ainsi en place, chaque Région musicale devra avoir une infrastructure administrative et technique auprès des directeurs régionaux des Affaires culturelles." 36

- Une volonté d'instituer des "relais de transmission" ${ }^{37}$ : ce schéma vertical de délégation ne dépareille pas du mouvement général de l'implantation du ministère en région, mais ne se plie pas non plus à celle-ci ; le placement des délégués régionaux auprès des D.R.A.C. n'a rien d'un passage de relais, par la direction de la musique, de l'animation musicale aux Directions régionales. La “désinvolture” des délégués à la musique à l'égard de l'autorité des D.R.A.C. que soulignera Jean-Luc Bodiguel ${ }^{38}$ laisse entrevoir que les délégations musicales seront avant tout, et pour longtemps, en relation directe avec leur direction centrale.

L'échelon régional de l'inspection ne vient pas davantage émanciper les régions musicales : si l'animation est placée, dans le plan de dix ans, sous le contrôle d'un“inspecteur

\footnotetext{
${ }^{34}$ Service de la musique, de l'art lyrique et de la danse, Plan de dix ans pour l'organisation des structures musicales françaises, 22 juillet 1969, p.2..

${ }^{35}$ Ibidem.

${ }^{36}$ Ibid.p.5. Titre III - Animation et contrôle.

37 "Pour bien gouverner, il faut, selon moi, être informé de tout et déléguer ses pouvoirs : des relais de transmission montant et descendant se révèlent vitaux pour une action à l'échelon d'un pays comme la France" Marcel Landowski, Batailles pour la musique, Paris, Seuil 1979, p.124.

${ }^{38}$ Jean-Luc Bodiguel, L'implantation du ministère de la culture en région, Naissance et développement des directions régionales des affaires culturelles, comité d'histoire du ministère de la culture, Paris, la documentation française, 2000, p.263.
} 
musical régional", lui-même en relation avec la direction de la musique, l'inspection musicale régionale ne sera finalement pas mise en place par Marcel Landowski, mais seulement en 1982, par Maurice Fleuret ${ }^{39}$.

\section{De l'animateur musical en région au délégué musical régional}

L'animateur musical devient, dans le plan de dix ans, "délégué musical". A ce changement de dénomination correspond aussi un changement, à courant de l'année 1971, de l'ensemble du personnel représentant la direction de la musique en région : les délégués musicaux régionaux nommés par Marcel Landowski ne sont pas issus du C.N.S.M., mais sont pour la plupart issus des grandes fédérations musicales, J.M.F., A Coeur Joie, Centres musicaux ruraux, etc. L'évolution de l'animateur-artiste vers l'animateur-pédagogue concerne à cette période l'ensemble de l'animation culturelle. Elle marque bien, concernant la direction des maisons de la culture, "la destabilisation d'une doctrine" secteur musical, un retour aux bases socio-éducatives de l'animation musicale.

Cette inflexion est accentuée par les conditions posées à l'accès au Fonds d'intervention culturelle mis en place en 1971, qui concernent les projets culturels à dimension éducative, et précipitent la création, par la direction de la musique, d'associations musicales régionales et départementales ${ }^{41}$ susceptibles de recevoir ces subventions et leur implication prioritaire dans les activités d'initation musicale. Ces associations constituent la première étape d'une décentralisation lente et contrôlée de la décision musicale, et le début de la montée en puissance de responsables musicaux recrutés sur place, non nécessairement muciens professionnels, mais militants de la musique et ayant le goût de l'administration territoriale.

Les régions les plus soutenues par la direction de la musique en termes de rénovation de structures de diffusion, Pays de Loire, Rhône-Alpes, Ile de France, Midi-Pyrénées, ProvenceCôte d'Azur, ou les régions les plus actives musicalement, comme l'Alsace et le Nord-Pas de Calais, sont également celles qui bénéficient du plus important soutien du fonds interministériel. ${ }^{42}$ Ce déséquilibre musical géographique doit être relativisé par la dynamique espérée à partir des régions les plus fortes pour que se réalise, petit à petit, l'égalité prévue dans le plan de dix ans.

Par sa politique de structuration régionale de la musique, Marcel Landowski anticipe l'inflexion, donnée à l'action du ministère de Jacques Duhamel vers le "développement culturel" qui intègre une acception de la culture décentralisés ${ }^{43}$ et réhabilite l'éducation et la connaissance pour l'accès de tous aux grandes œuvres, sans pour autant considérer la

\footnotetext{
${ }^{39}$ Cf Anne Veitl, Noémi Duchemin (Lefebvre), Maurice Fleuret, une politique démocratique de la musique, Comité d'histoire du ministère de la culture, La documentation française ,2000 p.129

${ }^{40}$ Philippe Urfalino, l'invention de la politique culturelle, Paris, Pluriel, 2004, p.87

${ }^{41}$ appelées A.R.D.I.M., A.D.A.M.A, A.R.D.A.M., A.D.D.I.M, A.D.D.M...suivant les régions et départements.

${ }^{42}$ Tableau de répartition des crédits du F.I.C. par Régions 1971-1980, in : Bertrand Castellani, A la marge de la politique musicale française, le Fonds d'intervention culturelle (1971-1981) p.24

43 “ C'est avant tout dans son milieu naturel, c'est-à-dire la ville, le département, la région, que l'exigence de culture s'enracine et se développe" annonce Jacques Duhamel à l'Assemblée nationale lors de l'examen et l'adoption du budget de 1972 (cité par Urfalino, op.cit.p.274.)
} 
possibilité de cultures musicales territoriales, qui renverrait à la culture telle qu'elle fut définie par Jacob Burckhardt comme « la somme des créations de l'esprit, nées spontanément et qui ne prétendent pas à une valeur universelle ni à un caractère obligatoire.» ${ }^{44}$ L'objectif “de donner au plus grand nombre possible de Français la musique de qualité à laquelle ils ont droit" ${ }^{45}$ interdit, il est vrai, d'envisager l'aléatoire et la diversité comme valeurs positives, et maintient la fonction performative d'un contrôle central de la décentralisation musicale.

\footnotetext{
44 "Kultur nennen wir die ganze Summe derjenigen Entwicklungen des Geistes, welche spontan geschehen une keine universale oder zwangsgeltung in Anspruch nehmen." (Weltgeschichtliche Betrachtungen, Berlin 1905, Kröner, Stuttgart, 1978, 496p., p.56 / Considérations sur l'histoire universelle, II. 3. "La Culture".)

${ }^{45}$ Note sur la musique, Rapport de Monsieur Landowski, Inspecteur général de l'enseignement musical, à l'intention de Monsieur Antoine Bernard, Directeur du Cabinet de Monsieur le Ministre des Affaires Culturelles, datée du 21 avril 1966. (A.N.19950514/24 -1)
} 